\title{
An integrated model for adolescent inpatient group therapy
}

\author{
D. GARRICK ${ }^{1}$ BSCN, RN \& C. EWASHEN ${ }^{2} \mathrm{MN}, \mathrm{RN}$ \\ ${ }^{1}$ Adolescent Outreach Therapist, Young Adult Program, Department of Psychiatry, Foothills Medical Center, \\ Calgary AB and ${ }^{2}$ Assistant Professor, Faculty of Nursing, University of Calgary, AB, Canada
}

Correspondence:

D. Garrick

Young Adult Program, SSB

Foothills Medical Center

1403-29 St, NW

Calgary

AB T2N 2 T9

Canada
GARRICK D. \& EWASHEN C. (2001) Journal of Psychiatric and Mental Health Nursing

$8,165-171$

An integrated model for adolescent inpatient group therapy

This paper proposes an integrated group therapy model to be utilized by psychiatric and mental health nurses; one innovatively designed to meet the therapeutic needs of adolescents admitted to inpatient psychiatric programs. The writers suggest a model of group therapy primarily comprised of interpersonal approaches within a feminist perspective. The proposed group focus is on active therapeutic engagement with adolescents to further interpersonal learning and to critically examine their contextualized lived experiences. Specific client and setting factors relevant to the selection of therapeutic techniques are reviewed. Selected theoretical models of group therapy are critiqued in relation to group therapy with adolescents. This integrated model of group therapy provides a safe and therapeutic forum that enriches clients' personal and interpersonal experiences as well as promotes healthy exploration, change, and empowerment.

Keywords: adolescents, group therapy, integrated model

Accepted for publication: 18 September 2000

\section{Introduction}

Historically, the practice of group therapy in inpatient settings evolved from a variety of theoretical models, the psychodynamic and the cognitive-behavioural being two of the most common. These differing models of group therapy are based on very different assumptions regarding the role of the group therapist, the desired relationships within the group, and the primary focus of therapeutic change. How then do clinicians, specifically nurse-therapists, understand the practice implications of different theoretical models, and become effective group therapists in their unique clinical settings? The ability to critically select and modify therapeutic techniques to best accommodate the conditions of practice becomes a valuable indicator of competent group skill development. In this regard, adolescent group therapy poses a unique challenge, as group practice models originate from the study of adult clinical practice. This paper proposes that nurses create an integrated group therapy model; one designed to meet the therapeutic needs of adolescents in an inpatient mental health setting. Specific client and setting factors that guide the selection of therapeutic techniques are reviewed. Select theoretical models of group practice are critiqued in relation to group therapy with adolescents. The resulting composite of approaches relevant to this population is then proposed as an integrated group therapy model.

\section{Clinical setting}

The context in which the group is conducted is very relevant when deciding the clinical appropriateness of various therapeutic models. For the purpose of this paper, the setting is an acute adolescent psychiatric inpatient unit in a large urban acute care hospital. The average length of stay for clients is four to six weeks. The adolescent program consists of a multidisciplinary team, although the medical model guides treatment, and decisions are 
ultimately made by the psychiatrist based on psychiatric presentation and diagnosis. To maintain a safe and contained environment for psychiatric treatment, a very structured behavioural management system governs the whole adolescent program. As part of this program, an open psychotherapy group is scheduled for one hour, three times each week. This group is practised from an interpersonal cognitive-behavioural perspective. Co-therapists facilitate the group, with five therapists rotating through this role. Therapists often have other treatment roles within the program (as psychiatrist, individual or family therapist) and come from a variety of academic backgrounds (medicine, psychology, social work and nursing). The intent of the current adolescent therapy group is to provide a supportive yet contained forum to share and address unit, treatment and individual issues. Creating an interactive sense of group cohesion and universality is a key therapeutic focus. The therapists assess levels of functioning within the group and facilitate members' participation, awareness of health issues and future planning, including preparation for discharge. Group sessions create therapeutic space for the adolescents to practise having a voice and to actively participate in their treatment and care. The impetus for proposing an alternative integrated group model arose from the writers' curiosity on how to expand the therapeutic space in adolescent groups so as to further the voice of adolescent members and more critically examine the context of their lived experiences, in and out of the group.

\section{Unique target population}

Adolescents admitted to the inpatient program range in age from 13-18 and are heterogeneous in terms of diagnostic presentation. Diagnoses include schizophrenia, major depression, bulimia, anorexia nervosa, bipolar disorder, pervasive developmental delay, obsessive compulsive disorder and post-traumatic stress disorder. Adolescents are pre-screened for willingness to participate in group and may still present with acute symptoms while a group member. Many clients have limited ego strength, are susceptible to narcissistic injury, and are therefore a vulnerable population.

\section{Developmental considerations}

It is important to bear in mind the clients' developmental stages when considering various group techniques. Piaget's theory of cognitive development suggests adolescents develop towards more abstract formal cognition from a stage of concrete operational thinking, while psychosocially, according to Erikson's model, adolescents struggle with issues of autonomy, identity, authority, independence, role clarification and peer relations (Townsend 2000). Adolescents, when initially admitted to the unit, often present as developmentally younger than their chronological age would suggest. It is crucial to consider the target adolescent population as well as developmental and contextual factors in order to make appropriate group therapy modifications, since most group models originate from the study of adult practice and thus may expect and interpret behaviours according to adult norms.

For the purpose of this paper, the psychodynamic, interpersonal and cognitive-behavioural group therapy models are reviewed, as they are prominent models of practice in the clinical setting. The group-as-a-whole and feminist models are also selected as possibilities for group work with adolescents, given the focus on membership responsibility which is fitting with adolescent peers, and on the members' contextualized lived experiences, respectively. The following critique details the rationale for the suggested appropriateness of each model as applied to the adolescent population.

\section{Critique of theoretical models}

\section{Psychodynamic model}

Rutan's psychodynamic model relies heavily on the principles of psychoanalytic theory (Rutan \& Stone 1984, Rutan 1992). It assumes that maladaptive behaviours and beliefs are a result of unconscious drives and conflicts. According to Rutan (1992, p. 20), the goal of therapy is 'to help the patient gain awareness of those parts of the unconscious that result in destructive distortions in present-day perceptions.' Rutan adds that the therapist traditionally takes a very neutral, inactive stance, allowing free association among group members so that various unconscious projections, distortions and transference issues will inevitably come forward. Only then does a therapist make an interpretation to facilitate members' consciousness of and ability to correct these maladaptive patterns.

Practising psychoanalytic work in the inpatient adolescent setting proves difficult as this therapy is long-term in nature and the therapist is relatively inactive. Piper et al. (1992, p. 66) suggest that for inpatient group work 'the therapist simply can't afford to be passive. However, maintaining an active presence, which we refer to technically as 'consistent focusing' runs the risk of violating certain technical principles that are often associated with the psychoanalytic orientation.' The psychoanalytic stance with adolescents often provokes strong reactions around authority, complicating more immediate treatment issues. The psychoanalytic goal, that group members become con- 
scious of previously unconscious distortions and gain insight into these distortions, is unreasonable within a short hospital stay and developmentally, the adolescents may have yet to achieve sufficient abstract thinking abilities.

A review of the literature confirms the inappropriateness of psychoanalytic therapy with adolescents due to developmental factors (Lewis 1993). In fact, Anna Freud (as cited in Lewis 1993, p. 345) said 'One cannot analyse in adolescence. It is like running into a freight train.' If psychoanalytic work is questionable for adolescents in general, such group practice is especially incongruent with the vulnerabilities of adolescent inpatient psychiatric clients. Inpatient clients present with minimal ego strength and are especially vulnerable given the psychological, emotional, and cognitive decompensation that may already exist due to psychiatric illness (Rice \& Rutan 1987). The therapist must be more verbally active, genuine in affect, and not readily challenge an adolescent's defences or selfrepresentations (Jacobs 1996). A psychoanalytic therapeutic stance with these clients is further complicated by role inconsistencies as the group therapists often interact with the adolescents in other roles within the overall program milieu. For example, a nurse therapist may be providing both family and individual therapy for an adolescent on the unit who is also a member of the group.

In conclusion, there is a clear lack of fit between the cognitive, psychosocial and psychiatric vulnerabilities of the inpatient adolescent population and the therapeutic stance and focus of the psychoanalytic group model. To work with adolescents means having to 'violate the principles of abstinence, neutrality, and primary reliance on interpretation' (Jacobs 1996, p. 233), principles vital to psychoanalytic work. The adolescents receiving psychiatric inpatient treatment demand a more directive, supportive and contained therapeutic space. The goal of attempting to make conscious those unconscious patterns and distortions that become evident in the psychoanalytic group process can only occupy a very limited and selective space in adolescent inpatient work.

\section{Interpersonal model}

Yalom's $(1985,1995)$ interpersonal model of group practice is rooted in the theoretical assumption that there is a basic 'human drive for interpersonal attachment and the maintenance of self-worth within interpersonal terms' (Leszcz 1992, p. 38). When distorted assumptions are made about the self from past interactions, it may result in maladaptive beliefs and interpersonal behaviours. The goal in Yalomian interpersonal therapy is to create a therapeutic space in which distortions and maladaptive interaction patterns can be brought to the fore, so members can experience responses from others through corrective emotional experiences that further healthy interpersonal learning. The therapist, through role modelling and facilitating group cohesion, norm setting, and an emotionally containing environment, provides opportunities to reflect on member interaction patterns. The therapist role is relatively transparent, with frequent use of self-disclosure and purposeful demystifying of one's expert position. 'The therapist in this model of group therapy will be very active but he will be active in the interest of stimulating activity on the part of group members and with the aim of making the group less rather than more leader dependent' (Leszcz 1992, p. 56).

Given the adolescent struggle for autonomy, difficulty with authority, and focus on peer relations, this therapeutic position provides the needed structure, and focus on autonomy, independence and interpersonal interactions. Church (1994, p. 107) reviews a study that explores various therapeutic stances in adolescent groups, and found that adolescents:

'responded more favourably to an egalitarian relationship rather than an authoritarian one. It seems important therefore for therapists to be cognizant of the role of autonomy in shaping the therapeutic relationships with adolescents and to recognize adolescents' desire to work out their own directions as well as their need for connection and structure.'

The focus on interpersonal here-and-now interactions does fit with the intent of the adolescent inpatient group discussed in this paper. However, the interpersonal group model is a long-term therapy and requires time to establish group culture and norms, and to allow distortions to come forward and be worked through therapeutically (Yalom 1995). Such time is a luxury that does not exist in inpatient settings. Additionally, these adolescents, given their acute mental state, often have minimal ego strength and are at risk of narcissistic injury. To challenge interpersonal distortions too quickly may be harmful, in that the adolescents' jeopardized sense of self may be quickly defended, exacerbation of symptoms may occur, and the group forum rendered therapeutically unsafe for that individual.

The general principles guiding the therapeutic stance and interpersonal focus of this model hold possibilities for group work with an adolescent population. However, the factors intrinsic to the acute care setting and psychiatric client base demand modifications of techniques from the pure interpersonal model. The concerns of high client turnover, brief duration of treatment, and the range and acuity of psychopathology complicate traditional interpersonal group practice for psychiatric inpatients. Yalom 
(1995) suggests altering the goals of interpersonal group in this context to include engaging members in the therapeutic process, facilitating cohesion and a sense of universality and altruism, identifying problems for future work, decreasing isolation, and reducing the anxiety around hospitalization. Yalom advises that for inpatient group work, a therapist adjusts technique and perceives the life of the group as one session. He or she must 'activate the group and call on, actively support, and interact personally with members' (Yalom 1995, p. 463). Therapists should provide structure, focus on the here and now, identify interpersonal concerns, reinforce strengths, and encourage continued work within other forums.

According to Yalom (1995, p. 472), a therapist must 'structure the group and yet, at the same time, avoid infantilizing its members.' He adds (p. 478), 'the leader must structure the group so as to encourage each member's autonomous functioning.' The concerns of practising a pure interpersonal model with adolescent inpatient clients are remedied with the modifications articulated by Yalom. This reformulated inpatient interpersonal approach provides a valuable guide for adolescent inpatient group therapy and acknowledges the unique client and contextual factors.

\section{Group-as-a-whole model}

According to Wilfred Bion's (1959) model, the therapist takes a position on the boundary of the group, not interacting with individual members but rather observing the whole group process, in practising group-as-a-whole. A basic assumption of this model is that individual functioning and the meeting of needs is revealed through the whole group functioning in relation to the group leader. The therapist only makes comments that address the groupas-a-whole dynamics. Various projections and group assumptions become evident over time and the therapist provides interpretations of the whole group process (Rioch 1972).

In considering the application of this model to adolescent inpatient settings, the length of time of therapy again proves problematic. This group model, while therapeutically addressing issues related to adolescents' developmental drive for autonomy and independence, requires a longer term therapy contact. The time needed to create group culture and cohesion and work through various projections and group assumptions is limited in inpatient settings.

Church (1994), in reference to adolescent psychotherapy groups, advocates that therapists support member autonomy, balanced with connection, containment, and an egalitarian culture. Further modification in approach is necessary with psychiatrically ill adolescents. It is impor- tant that therapists are aware of each group member's mental state, ego strength, and vulnerability. Soo (1992, p. 79) supports this by saying:

'The attenuation of permissiveness and the amount of structure necessary is established by the group therapist from his diagnostic understanding, the age appropriateness of his members, the balance of the membership, and the treatment goals of his group.'

Soo stresses the need for structure with psychiatrically vulnerable youth, suggesting group treatment of an unstructured or permissive nature is contraindicated with adolescents of compromised ego strength. Group work that is too unstructured may actually exacerbate symptoms such as psychotic behaviour.

Due to these concerns, group-as-a-whole practice seems inappropriate for nurse therapists in an adolescent psychiatric inpatient program. The permissive and unstructured format of group-as-a-whole, given the acuity and high turnover of clients, risks lack of emotional containment and possible regression and narcissistic injury to adolescents under care. Additionally, the permissiveness of the group-as-a-whole approach encourages adolescent selfgovernance that may conflict directly with the hierarchical, structured inpatient program milieu. Adolescent confusion and anger may arise due to mixed messages. Splitting may also occur between the group-as-a-whole and unit philosophies. This suggests limited and judicious use of group-asa-whole therapy with an inpatient adolescent population.

\section{Cognitive-behavioural therapy}

Cognitive-behavioural group therapy is typically time limited and focuses on specific problem areas or diagnostic groups. A rationalist cognitive model assumes that people develop cognitive misconceptions that may evolve into irrational beliefs as to how they perceive themselves, others and their world (Wessler \& Hankin-Walker 1989, Corey 1995). Rigid adherence to unrealistic, absolute expectations may result in 'dysfunctional thoughts (e.g. obsessions), feelings (e.g. panic, depression and selfhatred), and behaviours (e.g. phobias and compulsions)' (Ellis 1992, p. 64). Treatment focuses on identifying dysfunctional core cognitions as well as plans and actions to change them. The therapist intervenes with individuals in a group setting under the assumption that members are in the group to change. The therapist actively challenges dysfunctional cognitions through engaging members in various exercises and assigning homework to assist members' understandings of the connections between thoughts, feelings and behaviours.

How appropriate would this approach be for adolescent inpatient groups? The assumption that members want to 
change cannot be generalized to psychiatric inpatients, let alone many other groups. The client heterogeneity and acuity of psychiatric concerns complicate use of this model. Many clients do not consent to treatment and resist change, in part, due to acute psychopathology. Even if ready to contemplate other choices, the therapeutic stance with this model seems inappropriate for adolescents. Inpatient adolescents are too psychiatrically vulnerable to tolerate direct challenges. The therapist's high level of activity and use of confrontation may create interactions that are too highly stimulating. In a study of adolescents' responses to different therapeutic stances, Church (1994, p. 107) advocates for

'[An] approach being analogous to authoritativedemocratic parenting ... The adolescents responded more positively to the egalitarian, nonauthoritarian relationship than to the directive one. It also appears that they wanted some structure and guidance ... but they wanted to initiate the asking, not have advice imposed on them.'

In this sense, pure cognitive-behavioural therapy is too direct for adolescent inpatient practice given the risk of potential power struggles with perceived authority figures. Cognitive-behavioural approaches are more valuable when the therapist helps members consider change from a more supportive, less directive therapeutic stance. Cognitivebehavioural strategies are especially useful when the group members are healthy enough to explore and practice ways such as preparation for discharge.

\section{Feminist theory}

To practice feminist therapy means understanding a broader theoretical perspective. Key to feminist practice is 'the concept of 'gendering', that is, being shaped or defined by society's structures and assumptions about gender' (Reed \& Garvin 1996, p. 17). Also central to this perspective, according to Reed \& Garvin (1996), is exploring 'the dynamics of how class, race, ethnicity, sexual orientation, and other factors linked to societal inequities intersect with, and change, our understanding of gender-based inequities and gender processes'. In therapy, this translates to group consciousness-raising regarding relations of power, oppression and privilege. Discussion focuses on societal/political biases related to gender, age, race, class and ethnicity, and the impact on a client's sense of self, and their lived experiences. A process of consciousness-raising is valued as a means to group and social change. A feminist therapist assists group members to understand personal changes from the perspective of society, culture, and context (Lazerson 1992). Feminist therapy facilitates understanding the effects of power and ways to utilize relations of power positively in one's life (Bender \& Ewashen
2000). The power of language is explored in terms of using more liberating and empowering language. In feminist practice, there is a constant effort to connect, validate and integrate, rather than polarize, different experiences. The multitude of ways individuals learn and understand their experiences is valued.

How can the feminist perspective enhance therapeutic practice with adolescent psychiatric inpatients? It is clear that the principles of feminist practice have the potential to make public the private oppressions these adolescents experience. Anderson (1997, p. 1) comments that 'females and males under age 18 often have been assumed incapable or unworthy of having inherent power, human rights or valid needs, either by adult decision or default'. If such oppressions exist for adolescents in general, they are compounded for those adolescents in psychiatric treatment. They are in treatment often without their consent and are treated within a medical model that is guided by diagnostic labels. In such treatment, according to McGraw Schuchman (1997): 'interventions are constructed or adapted from traditional models of therapy which are limited in their understanding of adolescent needs', and 'include: the therapist in the role of expert, that direction for interaction and treatment is not placed with the client, a propensity to rely on diagnostic categorization rather than focus on client experiences, and the problem with access and flexibility in professional services' (pp. 102-103). A nurse practising therapy from the feminist perspective openly speaks about and critically re-examines the role of power in group, in treatment and in the context of the members' lived experiences.

Adolescence is a stage in which individuals from lasting constructs regarding how they value themselves and understand their ability to master developmental change in their lives (Rice, 1996). They are vulnerable to internalizing societal biases and oppressions due to age, gender, class, and ethnicity. Adolescent psychiatric inpatients are at an even greater risk of being immobilized within an oppressive and disempowered sense of self. McGraw Schuchman (1997, p. 103) states that feminist theory has:

'constructs which guide the practice of therapy such as building on competencies, recognizing power imbalances, using a growth (vs. illness) model of therapy, developing a sense of community, emphasizing autonomy and self-direction, challenging therapeutic distance, and utilizing self-disclosure in helping relationships.'

This group approach validates client and group experiences, connects members with a sense of universality and enables healthier perspectives that empower adolescents to be active agents of change in their lives. This suggests that not just nurses, but all therapists, reflect on the messages 
their therapeutic approaches convey to clients in order to ensure oppressions and biases are not reinforced.

The feminist perspective provides clients with a more validating, empowering, contextual understanding of their circumstances. This is lacking in traditional models that risk pathologizing clients by localizing exploration to 'their distorted' interactions, assumptions and cognitions. At the very least, this approach creates therapeutic space for adolescents to dialogue about issues of power and position from a societal/political context, facilitating a healthier understanding of their circumstances and empowering possibilities for change. The feminist perspective, or lens, expands the therapeutic value of adolescent inpatient group therapy.

\section{Summary of the integrated group therapy model}

This paper proposes a model of group therapy for adolescent psychiatric inpatient that is primarily comprised of interpersonal approaches within a feminist perspective. Group-as-a-whole, psychoanalytic and cognitivebehavioural models seem less applicable to adolescent inpatient care, given the implications of the psychiatric vulnerabilities of the clients and factors intrinsic to the setting (client turnover and program structure). Addressing unconscious conflicts or drives and recognizing distorted cognitions and group assumptions may be more useful in unique group circumstances and in longer term therapy situations. The nurse-therapist must be judicious in the use of these models.

Yalom's interpersonal model of group therapy provides the most fitting therapeutic stance for group therapy with adolescent inpatients (Yalom, 1995). This model acknowledges the need for greater structure and containment because of the acute psychopathology of clients, and additionally values interpersonal group work in building cohesion and a sense of universality. The interpersonal approach appreciates the need to focus on problem identification from an interpersonal perspective, addresses issues and feelings concerning treatment, and empowers members to be actively involved with each other in the process of their treatment and therapy. This includes bridging of the interpersonal work into the larger treatment milieu.

However, there still exists a gap in addressing the significance of the client's understanding of their lived experience. This gap is adeptly addressed by practising group therapy within a broader feminist lens. With the application of a feminist perspective, the clients' sense of their own lived experiences is enriched through exploring the influences of power, position, cultural biases and the societal/political context. The benefit to the client is a more validating and empowering therapeutic experience. As a nurse-therapist working with adolescents in a group setting, it is important to increase a client's sense of independence, self-directedness, and empowerment in managing their circumstances. It is equally important not to pathologize or limit client understanding of their circumstances or contribute to internal dysfunction, as is often implied in psychiatric treatment.

Practising group therapy from a feminist perspective and constantly reflecting on these issues safeguards against such counterproductive effects. By nurse-therapists thoughtfully drawing from this integrated model of adolescent group therapy, the potential for an optimum group therapy experience is achieved; one that is congruent with the uniqueness of the clients and the particular demands of inpatient settings. This integrated model of group therapy provides a safe and therapeutic forum that enriches clients' personal and interpersonal experiences as well as promoting healthy exploration, change and empowerment.

The question of the possibilities to practice this proposed integrated model in the adolescent inpatient setting still remains. The writers contend that the proposed integrated model of feminist theory and Yalomian group therapy theory enriches adolescents' understandings of their lived experiences by exploring the influences of power and bias within societal/political contexts, and by providing a more validating and empowering therapeutic experience. However, applying this model to practice raises a number of questions for consideration:

1 What are the comfort levels of group therapists when practising from a feminist stance?

2 How open is the group therapy team to addressing the influences of power, position and bias in the group, in the treatment program and in the clients' other lived experiences?

3 How tolerant is the treatment team, as part of the established hierarchical structure of inpatient care, to a therapeutic forum to discuss such influences?

4 Could, and would, the multidisciplinary team support a group that addresses the influence of power, position and bias on the clients' experiences, including while in care?

5 Can a hierarchically structured program milieu and the proposed group approach coexist and support each other and still maintain the therapeutic integrity of the overall program?

In keeping with feminist beliefs, asking such questions and reflecting on the influences of power, privilege and the societal/political context becomes an integral part of the change process. Such discussion creates space for considering how to further enrich therapeutic group experiences for adolescents. 


\section{References}

Anderson G. (1997) Introduction: children, adolescents and their powerholders in therapy settings. Women and Therapy 20,1-6.

Bender A. \& Ewashen C. (2000) Group work is political work: a feminist perspective of interpersonal group psychotherapy. Issues in Mental Health Nursing 21, 297-308.

Bion W.R. (1959) Experiences in Group. Basic Books, New York. Church E. (1994) The role of autonomy in adolescent psychotherapy. Psychotherapy 31, 101-108.

Corey G. (1995) Theory and Practice of Group Counseling, 4th edn. Brooks/Cole, Pacific Grove, CA.

Ellis A. (1992) Group rational-emotive and cognitive-behavioral therapy. International Journal of Group Psychotherapy 42, 63-80.

Jacobs T. (1996) On therapeutic interventions in the analysis of certain 'unanalyzable' patients. Contemporary Psychoanalysis $32,215-235$.

Lazerson J. (1992) Feminism and group psychotherapy: an ethical responsibility. International Journal of Group Psychotherapy 42, 523-546.

Leszcz M. (1992) The interpersonal approach to group psychotherapy. International Journal of Group Psychotherapy 42, 37-62.

Lewis O. (1993) Adolescence, social development, and psychotherapy. American Journal of Psychotherapy 47, 344-352.

McGraw Schuchman K. (1997) Feminist approaches to working with adolescents: acknowledging competencies and developing alternative interventions. Women and Therapy 20, 101-110.

Piper W.L., McCallum M. \& Azim H.F. (1992) Adaptation to
Loss Through Short-Term Group Psychotherapy. The Guilford Press, New York.

Reed B.G. \& Garvin C.D. (1996) Feminist thought and group psychotherapy: Feminist principles as praxis. In: Women and Group Psychotherapy (ed. De Chant B.), pp. 15-49. Guilford, New York.

Rice F.P. (1996) The Adolescent: Development, Relationships \& Culture, 8th edn. Allyn \& Bacon, Boston.

Rice C.A. \& Rutan J.S. (1987) Inpatient Group Psychotherapy: a Psychodynamic Perspective. Macmillan, New York.

Rioch M. (1972) The work of Wilfred Bion on groups. In: Progress in Group and Family Therapy (eds Sayer C.J. \& Kaplan H.S.), pp. 18-32. Brunner/Mazel, New York.

Rutan J.S. (1992) Psychodynamic group psychotherapy. International Journal of Group Psychotherapy 42, 19-35.

Rutan J.S. \& Stone W.N. (1984) Psychodynamic Group Psychotherapy. Macmillan, New York.

Soo E.S. (1992) The management of resistance in the application of object relations concepts in children's and adolescents' group psychotherapy. Journal of Child and Adolescent Group Therapy 2, 77-92.

Townsend M.C. (2000). Psychiatric Mental Health Nursing: Concepts of Care, 3rd edn. F.A. Davis, Philadelphia.

Wessler R.L. \& Hankin-Walker S. (1989) Cognitive group therapy. In: Comprehensive Handbook of Cognitive Therapy (eds Freeman A., Simon K., Beutler L. \& Arkowitz H.), pp. 559-581. Plenum Press, New York.

Yalom I.D. (1985) The Theory and Practice of Group Psychotherapy, 3rd edn. Basic Books, New York.

Yalom I.D. (1995) The Theory and Practice of Group Psychotherapy, 4th edn. Basic Books, New York. 